\title{
Research topics in regulatory science: Developmental and reproductive toxicity of human pharmaceuticals
}

\author{
Shin-ichi SEKIZAWA ${ }^{1 *}$ \\ Department of Veterinary Pathophysiology and Animal Health, Graduate School of Agricultural and Life Sciences, The University of Tokyo, \\ 1-1-1 Yayoi, Bunkyo-ku, Tokyo 113-8657, Japan
}

\begin{abstract}
In this review, I have discussed current issues related to risk assessments of developmental and reproductive toxicities during the use of pharmaceuticals in pregnant women or women of childbearing potential. Owing to the lack of empirical information on the use of pharmaceuticals (including those approved for the market) in pregnant women, the toxicity of these drugs has been generally evaluated in animal studies. In the case of embryo-fetal developmental (EFD) toxicity, in particular, testing of small-molecule pharmaceuticals in animals (namely rodents and rabbits) is required. The body of research in animal studies affords us an opportunity to explore numerous topics in regulatory science research. However, there are three major issues: risk communication, use of animals, and reduction or deferral of EFD toxicity studies for the development of human pharmaceuticals. Although regulatory science research may not be necessary for some topics, it would contribute to the reduction or replacement of testing in animals. It is hoped that regulatory science research in this field will proceed rapidly, with the goal of safe use of pharmaceuticals for women.
\end{abstract}

Key words: 3R principle, embryo-fetal developmental toxicity study, drug, Japan, pregnancy

\section{Highlights}

1. In this review article, I present possible topics of regulatory science in developmental and reproductive toxicity assessment of pharmaceuticals intended for human use.

2. In developing pharmaceuticals for human use, risk communication between physicians and patients is always an important consideration, as is the selection of appropriate species for animal studies, if needed, as well as the reduction or deferral of EFD toxicity studies.

3. Regulatory science research suggested here can ultimately contribute to implementation of the " $3 R^{\prime \prime}$ principles: the reduction, refinement, and, possibly, replacement of animal studies in drug development.

\section{Introduction}

Pharmaceuticals, compared with food and cosmetics, exert potent and selective targeting of certain physiological and pathophysiological mechanisms in pathogens or in the whole body that dramatically change the physical condition of an individual, with the goal of improving the condition or curing the disease. The power of pharmaceuticals also means that they have certain side effects or toxicities, which are generally evaluated by toxicity (but not necessarily pharmacology) studies using animals. Before a drug can be officially approved by regulatory authorities, the candidate pharmaceutical is tested in animals and in humans under very limited condition; in other words, clinical trials. However, candidate pharmaceuticals are not normally tested in pregnant women before regulatory approval, meaning

*Correspondence to: Sekizawa, S.: a-ssekiz@mail.ecc.u-tokyo.ac.jp Received: May 22, 2020; Accepted: Jun. 26, 2020 that pregnant women subjects are not included or are expressly excluded from clinical trials once pregnancy is recognized. Furthermore, even after market authorization, many pharmaceuticals are rarely used during pregnancy. Therefore, developmental and reproductive toxicity studies using animals, such as embryo-fetal developmental (EFD) toxicity studies, are required for the development and market authorization of pharmaceuticals to predict possible adverse effects not only in pregnant mothers but also in the fetuses and infants. In fact, there have been several devastating incidents associated with reproductive health outcomes caused by pharmaceuticals in the past, including the thalidomide disaster in the late 50s to early 60s [1], which led to the testing of pharmaceuticals in pregnant animals, including rodents and non-rodents. On the other hand, there is a growing concern in society for animal welfare and a call to reduce the use of animals, refine experimental design, and replace animal studies with alternative studies; 
these three goals are known as the 3R principles [2].

Recently, the guidelines for developmental and reproductive toxicity evaluation of pharmaceuticals for human use has been greatly revised by the International Council on Harmonisation of Technical Requirements for Pharmaceuticals for Human Use (ICH) [3]. Although numerous small-molecule pharmaceuticals have been found to be teratogenic and may cause embryo-fetal death in EFD toxicity studies using either rodents (rats and mice) or rabbits [4], the new guideline still states that the EFD toxicities of small-molecule pharmaceuticals should be evaluated using both rodents and non-rodents. Accumulating scientific data appear to indicate teratogenic agents might be misclassified as non-teratogenic when evaluated with only one animal species (most likely a rodent species); however, several methods of evaluating toxicity are now suggested under certain situations to reduce animal use, in accordance with the $3 \mathrm{R}$ policy. The guideline, in fact, offers the use of alternative assays to EFD studies, such as in vitro, ex vivo, and non-mammalian studies under certain specific circumstances; however there are still no validated assays for the evaluation of reproductive toxicity. In addition, the guideline describes the principles of human risk assessment for pharmaceuticals but does not provide information on the potential of actual clinical usage. In the context of the labeling of drugs for pregnant women, my goal in this review is to describe possible approaches to detect the actual risk of developmental and reproductive toxicities in humans as well as to reduce animal use in research. I address three major topics in regulatory science studies: risk communication, selection of animal subjects for testing, and possible reduction or deferral of EFD studies in animals.

\section{Risk Communication}

The notion of "risk communication for EFD toxicities" has different meanings depending on the frame of reference; for example, when a pharmaceutical developer is considering a candidate drug whose pharmacological mechanism is expected to induce EFD toxicities and communicates this risk to the regulatory authorities, EFD toxicity studies may consequently not be performed because the risk communication has been deemed sufficient. This communication means that the EFD toxicity of a candidate pharmaceutical will most likely be observed in animals if the study is performed, which is also supported by the recently revised ICH S5 guideline [3]. If this candidate pharmaceutical is approved for marketing, this fact can be indicated in a package insert. For example, 'contraindication for pregnant women' is included in the labeling in many cases. This labeling appears to be reasonable for readers, as many drugs with this labeling are prescribed by physicians. This is a risk communication between regulatory authorities and medical doctors, and there is no question about the understanding of the contraindication. However, in the case of anticancer pharmaceuticals, risk communication in the labeling may not be clear enough for readers to understand its meaning, as several of these drugs are not contraindicated for pregnant women. As an example, Portrazza ${ }^{\circledR}$ injection (necitumumab (genetic recombination), Nippon Kayaku Co., Ltd., Tokyo,
Japan), an antibody that antagonizes epidermal growth factor receptor signaling, is a pharmaceutical for patients with metastatic squamous non-small cell lung cancer and used in combination with gemcitabine and cisplatin [5]. Although such treatment is not prohibited for pregnant women, in my personal opinion, the description in the label does not seem to be logical: "administration to pregnant women or women who may possibly be pregnant is allowed only when the expected therapeutic benefit outweighs the possible risks associated with the treatment". This description implies an incomparable comparison, namely benefit for the mother $v$ s. risk to the fetus. A similar description is found in the label of Lorbrena ${ }^{\circledR}$ tablets (lorlatinib, Pfizer Inc., Tokyo, Japan) [6]. The following are issues with vague labeling such as necitumumab (genetic recombination) as an example.

Firstly, as both gemcitabine and cisplatin are contraindicated for pregnant women, does it mean that necitumumab has a lower risk of EFD toxicity than these two drugs? If so, this should be clarified in a regulatory science study. Otherwise, owing to the vague labeling, one may consider the use of necitumumab (genetic recombination) for pregnant women with cancer, despite the contraindication. If the ambiguity in risk communication is due merely to inconsistent labeling, this problem can be easily corrected.

Secondly, in the labeling of necitumumab (genetic recombination) there is a description of risk communication between medical doctors and patients, along with the recommendation that contraception should be required for women of childbearing potential. Considering that the indicated patient population is typically over middle age, women of childbearing potential may not often use this pharmaceutical, and the labeling does not state what will likely happen if women of childbearing potential use this medication or whether termination of the pregnancy can be an option. This is a very difficult issue to be answered correctly (there is no perfect answer), although it may be beneficial for doctors to identify which pharmaceuticals have greater or lesser risks among those with similar pharmacology. This could be a topic in regulatory science studies. In fact, there have been studies comparing the EFD toxicities of pharmaceuticals with similar pharmacology. In one example, antiviral medications were compared. The EFD toxicity of Avigan ${ }^{\circledR}$ (favipiravir, Fujifilm Toyama Chemical Co., Ltd., Tokyo, Japan), an antiviral drug, was compared with that of the nucleoside analogs ribavirin and valaciclovir in rats [7]. Of course, each pharmaceutical has its own indication, meaning the target virus (es) and diseases are different, whereas nucleoside analogs have a certain spectrum of antiviral activities. Such toxicity data could be very informative for off-label information for pregnant women or women who may be pregnant during an emergency situation, such as the COVID-19 pandemic in 2020. In other pharmaceuticals, angiotensin receptor blockers (angiotensin II receptor antagonists), which are used for hypertension treatment, are contraindicated in pregnant women in Japan even without any clinical evidence (Table 1) [8-14]. Although it is difficult to conclude whether these pharmaceuticals exert direct adverse effects on fetal development (as their pharmacology could decrease blood pressure to the abnormal range in normotensive animals), 
it might be necessary to compare the EFD toxicities of angiotensin receptor blockers in hypertensive animals.

Thirdly, the risk of treatment during mid and late pregnancy is not apparent; for example, in the labeling of Portrazza ${ }^{\circledR}$ injection (necitumumab (genetic recombination)) [5]. Although the treatment can be used in women in early pregnancy, the labeling does not state whether the treatment has greater or less risk during mid and late pregnancy. Thus, medical doctors might not be able to advise patients and families of the possible risks in those periods. Clarifying this could be a subject of another regulatory science study. In another case, the labeling of Tecentriq ${ }^{\circledR}$ (atezolizumab; (genetic recombination), Chugai Pharmaceutical Co., Ltd., Tokyo, Japan) [15], a monoclonal antibody against protein programmed cell death-ligand 1, states that this pharmaceutical may cause miscarriages or still-birth based on its pharmacology and exerts possible adverse effects on the fetus due to its placental transfer. According to this description, this pharmaceutical may have slightly lower risk in the early stage of pregnancy than in the late stage, but it is unclear whether women who may be pregnant are at high risk or whether medication should be continued when pregnancy is recognized. Similar ambiguity can be found in the labeling of Norvasc ${ }^{\circledR}$ tablets (amlodipine besilate, Pfizer Inc., Tokyo, Japan) [16], which is, in fact, contraindicated for pregnant women or women who may be pregnant. However, its toxicity in animals was observed only in the late pregnancy period, causing prolonged pregnancy and parturition periods; moreover, there is no description about the EFD risks of this drug in the early pregnancy period. The EFD toxicities of calcium channel blockers, antihypertensives, all of which are contraindicated for pregnant women in Japan, are shown in Table 2 [16-23]. Some of these pharmaceuticals have no sign of critical and adverse effects in early pregnancy, thus there may be no need to prohibit the use of these pharmaceuticals in the first trimester or the first and second trimesters. This could be a subject of regulatory science research, unless there is enough safety evidence from studies of other pharmaceuticals with different pharmacology but in the same class i.e., many other antihypertensives are available for use with considerable efficacy and safety.

Breast cancer is the most frequently diagnosed cancer in Japanese women, with a five-year relative survival rate greater than $90 \%$ [24]. Included in this percentage are breast cancers associated with pregnancy. Pregnant women with breast cancer may have been exposed to or treated with anticancer pharmaceuticals, and thus sufficient data have been accumulated to generate a guideline for the chemotherapy treatment [25]. In this context, risk communication between medical doctors and patients is not mediated by labeling, but often by a guideline issued by a scientific society. In fact, the guideline for breast cancer treatments suggests several different strategies for treating patients who are pregnant (in the first trimester) and who are undergoing chemotherapy, (including tamoxifen); and one of the strategies is to consider pregnancy termination [25]. Regulatory agencies cannot specify "elective pregnancy termination" in the labeling of these pharmaceuticals, but the manufacturers of these drugs can choose to include product information that explains that termination is an option to consider. Furthermore, the labeling of pharmaceuticals for breast cancer treatment may have to contain information on fetal developmental risks upon exposure during the second and third trimesters. This could also be a labeling practice for other cancers, such as thyroid cancer, that have relatively high five-year relative survival rates and incident rates in patients of relatively young age. In the ICH S5 guideline [3], toxicities in pregnant women during the second and third trimesters are to be evaluated in pre- and post-natal developmental toxicity studies. However, the drug exposure of these studies normally includes the period of organogenesis, which corresponds to the first trimester of human pregnancy, and should have already been evaluated by EFD studies. Thus, in certain specific pharmaceuticals, such as those for breast cancer treatment, toxicity studies using pregnant animals 'after organogenesis' of the fetus may be useful in clinical usage if pre- and post-natal developmental toxicity studies, which are usually not performed on the development of anti-cancer pharmaceuticals, are required. An assessment

Table 1. Angiotensin receptor blockers contraindicated for pregnant women in Japan

\begin{tabular}{|c|c|c|c|c|c|c|c|}
\hline Drugs* & Azilsartan & Candesartan & Irbesartan & Losartan & Olmesartan & Telmisartan & Valsartan \\
\hline Labels** & Not included & Included & Not included & Included & Not included & Included & Included \\
\hline
\end{tabular}

*Each source of drug information is listed in the References [8-14]. ${ }^{* *}$ Contraindication is based on the Pharm/Tox information in humans using similar drugs except the drug indicated in the column heading (Not included) or including the drug indicated in the column heading (Included).

Table 2. Embryo-fetal developmental (EFD) toxicity of calcium channel blockers contraindicated for pregnant women in Japan

\begin{tabular}{lcccccccc}
\hline Drugs $^{\star}$ & Amlodipine & Azelnidipine & Benidipine & Cilnidipine & Diltiazem & Manidipine & Nicardipine & Nifedipine ${ }^{* *}$ \\
\hline EFD toxicity & $\begin{array}{c}\text { Prolongation } \\
\text { of the } \\
\text { parturition } \\
\text { period }\end{array}$ & $\begin{array}{c}\text { Embryo } \\
\text { lethality }\end{array}$ & Fetal toxicity & Fetal toxicity & $\begin{array}{c}\text { Fetal } \\
\text { malformation } \\
\text { and lethality }\end{array}$ & $\begin{array}{c}\text { Prolongation } \\
\text { of the } \\
\text { parturition } \\
\text { period }\end{array}$ & $\begin{array}{c}\text { Prolongation } \\
\text { of the } \\
\text { parturition } \\
\text { period }\end{array}$ & $\begin{array}{c}\text { Fetal } \\
\text { malformation } \\
\text { and toxicity }\end{array}$ \\
\hline
\end{tabular}

*Each source of drug/toxicity information is listed in the References [16-23]. **Nifedipine is contraindicated only for pregnant women at less than 20 weeks of gestation. 
of whether this kind of toxicity study-evaluating toxicity in the animal fetus after organogenesis and offspring - is much more valuable than the conventional pre- and postnatal developmental toxicity study, in which drug exposure starts at the beginning of organogenesis, could be a topic for further study in regulatory science.

Overall, regulatory science studies in this field can clarify the beneficial effect of pharmaceuticals for pregnant patients versus the probability of fetal risks and can help provide accurate information on EFD risks to physicians, patients, and families.

\section{Animal Species}

In terms of the genetic similarity with humans, there is no doubt that non-human primate species are better animal models than rodents for the evaluation of pharmaceuticals for human use. However, EFD toxicity studies of smallmolecule human pharmaceuticals are normally performed in two animal groups, namely rodents and rabbits, instead of non-human primates. If non-human primates are used for EFD studies, testing with an additional animal species might not be needed, even if this could contribute to reduction of the $3 \mathrm{R}$ principles. In other words, the information obtained from EFD studies with rodents and rabbits is as significant as that obtained from studies with non-human primates, and that the replacement can greatly contribute to implementation of the $3 \mathrm{R}$ principles. This is not the argument I am making here, but, rather, I suggest that this is a question in regulatory science - if the teratogenicity of some pharmaceuticals cannot be detected by EFD studies in rodents and rabbits, which have successfully shown embryo-fetal malformation and lethality caused by human teratogens so far [26].

The requirement of EFD studies in rodents and rabbits for the development of pharmaceuticals for human use derives from the thalidomide tragedy that occurred in (West) Germany, the United Kingdom, Japan, and other countries in the late 50s [1]. Thalidomide does not cause teratogenicity in rodents, but it does in rabbits. The EFD toxicity of substances other than pharmaceuticals have been usually tested in studies with rodents (typically rats). As almost one tenth of pharmaceuticals do not exert teratogenicity in rats, but do in rabbits, or vice versa [4], some non-pharmaceutical molecules that do not show teratogenicity in rats or mice may be teratogenic in rabbits. It is unknown how many molecules or food ingredients could be teratogenic. This could be a topic of regulatory science studies, even though these substances are not as biologically active as pharmaceuticals. (Possibly, in vitro or other assays capable of predicting EFD toxicity in rabbits may be available in the future.) Because of the amount of historical data on EFD studies in rats, this species is more frequently used than rabbits in EFD toxicity studies. However, if EFD toxicity - not limited to malformation and embryo-fetal lethality - were found in rabbits but not in rats, might it be more appropriate that the pre- and post-natal developmental study is carried out using rabbits? This question could be discussed in an expert group of regulatory science studies.

Although the mechanism of the teratogenicity of thalido- mide has not been fully elucidated, a primary target has been identified, namely the protein cereblon [27]. This protein is also targeted by the thalidomide analogs lenalidomide and pomalidomide [28]. Interestingly, pomalidomide does not show teratogenicity in zebrafish and chickens [29], but does in rats and rabbits [30], whereas lenalidomide does not lead to malformations in the offspring of rats or rabbits but does cause malformations in the offspring of non-human primates [31]. Taken together, thalidomide and its analogs, all possibly targeting cereblon, cannot consistently exhibit their teratogenicity in one specific animal species [27, 30, 31]. Although the precise mechanisms are still unknown, immunomodulatory effects and intracellular signaling pathways differ greatly among animal species, including humans, and this may be the cause of the inconsistency in teratogenic effects. This suggests that extra caution may be required when examining the EFD toxicity of pharmaceuticals with immunomodulatory or immunotoxic effects in rodents and rabbits, and this should be clarified by regulatory science studies in the future. Moreover, cereblon can bind to large-conductance calcium-activated potassium channels (also known as BK channels) and thus may affect neuronal activity and development $[32,33]$. This is definitely an off-target effect of thalidomide and its analogs, but the possible side effects have not been detected by animal studies, such as EFD toxicity studies. However, it cannot be concluded whether thalidomide has no adverse effects on neuronal development, as there is no validated method to detect such adverse effects using animals. Although this area can be a field of future regulatory science research, a novel approach might be needed to perform a truly relevant risk assessment.

Some pharmaceuticals show considerable effects on the physiological condition of certain animals; for example, several antibiotics can eliminate to a large degree the intestinal microbiota of rabbits, resulting in poor health condition and weight loss. Therefore, in the development of antibiotics, EFD studies in rabbits have often been performed at low dose ranges - for example, in a rabbit EFD study for tebipenem pivoxil, the multiple exposure to humans was 0.7 for Cmax and 0.4 for AUC [34]- or have been canceled after pilot data are obtained. In the latter case, mice have frequently been used instead of rabbits in EFD studies. Six example antibiotics are presented in Table 3; most of these show the same maximum doses in both rats and mice, indicating similar maternal toxicities in both animals. In contrast, the maximum doses in rabbit studies are similar to the human doses in many cases, or far less in the case of amoxicillin (Table 3) [34-39]. In this context, two questions come to mind: 1) whether EFD risk assessments in mice are worth performing in addition to those in rats, as rats and mice are both rodents, and 2) whether EFD studies in rabbits should be performed or are worth performing if high exposure margins; i.e., the ratio in Table 3, can be reached in the study. These questions warrant further investigation in regulatory science studies.

With respect to biologics, especially monoclonal antibodies, most reproductive toxicity studies have been performed in non-human primates because of their pharmacology. Currently, EFD toxicities have been evaluated in extended 
Table 3. Highest doses $(\mathrm{mg} / \mathrm{kg}$ ) in embryo-fetal developmental (EFD) studies and maximum recommended human doses of antibiotics

\begin{tabular}{lcccccc}
\hline \multicolumn{1}{c}{ Drugs $^{*}$} & Amoxicillin & Azithromycin & Cefazolin & Clofazimine & Ertapenem & Tebipenem \\
\hline Rat & $1,000(5)$ & $200(20)$ & $2,000(20)$ & $50(12.5)$ & $700(11.7)$ & $1,000(250)$ \\
Rabbit & $10(0.05)$ & $40(4)$ & $125(1.3)$ & $15(3.8)$ & $30(0.5)$ & $3(0.8)$ \\
Mouse & $1,000(5)$ & $200(20)$ & $4,000(40)$ & $50(12.5)$ & $700(11.7)$ & $1,000(250)$ \\
Human & 200 & 10 & 100 & 4 & 60 & 4 \\
\hline
\end{tabular}

*Each source of drug information is listed in the References [34-39]. ${ }^{*}$ Calculated as $50 \mathrm{~kg}$ of human body weight. The number of parentheses indicates the ratio to human doses shown in the bottom of this table.

pre- and post-natal developmental studies using non-human primates instead of separate EFD studies, contributing to reduction of animal use. As many monoclonal antibody pharmaceuticals target and modulate the immune system, animals other than primates are often not physiologically or pharmacologically relevant; thus, rodents (or rabbits) have not been used in toxicology studies for the development of these pharmaceuticals. Although there have been numerous immunological experiments/studies using murine immune systems (approximately 400,000 publications in 2020 appear in PubMed with two search words, 'mice' and 'immunology'), many studies have been focused on creating humanized immune systems in mice under certain conditions [40-45]. During pregnancy, the female immune system can change to a large degree from that in the non-pregnant condition. It is still unknown whether 'humanized' mice would show similar immune responses during pregnancy to those of humans, and whether these mice could be a useful animal model for EFD toxicity studies. However, this may be a topic of regulatory science that can contribute to the replacement of animals, by using those mice instead of non-human primates or a combination of rats and rabbits, in accordance with the $3 \mathrm{R}$ principles.

\section{Reduction or Deferral of EFD Toxicity Studies for the Development of Human Pharmaceuticals}

The guideline for non-clinical (non-human) safety studies prior to human clinical trials for pharmaceuticals ( $\mathrm{ICH}$ M3(R2)) includes two separate statements pertaining to two different geographic regions [46]. One guideline states that in the United States, the assessment of embryo-fetal development can be deferred until before phase III clinical trials for women of childbearing potential using precautions to prevent pregnancy; i.e., using strict contraceptive methods. The other guideline states that in the European Union (EU) and Japan, definitive EFD toxicity studies should be completed before exposure to women of childbearing potential even in phase I clinical trials, unless preliminary EFD toxicity data are available from two animal species and precautions to prevent pregnancy during the clinical trials are used. This guideline would enable the inclusion of women of childbearing potential (up to 150 women) into investigational clinical trials for a relatively short duration (up to 3 months). Basically, this number and duration are derived from the guideline ICH M3(R2), in which the survey suggested that among 150 women of childbearing potential, nobody should become pregnant during three months of controlled clinical trials [46]. Nevertheless, it is clear that there is a regional difference, but what causes this difference? Although there is no explanation in the guideline, this difference might be related to the thalidomide tragedy, which occurred prominently in Europe and Japan, but rarely in the US. Until now, there has not been any signals of adverse events related to pregnancy during the early phases of clinical trials in the US. Based on this situation, regulatory agencies other than those of the US may need to investigate how many women have been pregnant and the outcomes of these women during phase I and II clinical trials with or without serious precautions to prevent pregnancy. Thus, this is clearly an important topic for future regulatory science studies in Japan and the EU. If data indicate that women of childbearing potential did not become pregnant or they were capable of strictly following the rule of clinical trials, such as contraception use, definitive EFD toxicity studies can also be deferred until before phase III clinical trials in countries other than the US, contributing to an overall reduction in animal use (if sponsors decide not to proceed into phase III programs, EFD studies are unnecessary).

The new ICH S5 guideline [3], has introduced a new concept: in certain circumstances, properly qualified alternative assays, including in vitro and non-mammalian in vivo assays, have the potential to defer or replace conventional in vivo studies (EFD toxicity studies), and several scenarios are presented in the annex of the guideline. Among them, one scenario is for "pharmaceuticals expected to be embryo-fetal toxicants" and another is for "pharmaceuticals intended to treat severely debilitating or life-threatening diseases". Both scenarios may sometimes be suitable for some specific pharmaceuticals, such as anticancer agents. In such cases, one could argue which scenario should be taken if drug developers and sponsors have qualified alternative assays. However, considering the strategies in each scenario [3], the latter may potentially decrease the number of EFD studies (only one EFD study is required), thereby contributing to implementation of the $3 \mathrm{R}$ principles (see Fig.1). In both scenarios, more EFD studies are required to declare a given pharmaceutical negative for malformation and embryo-fetal lethality, but once a study or assay shows positive results, no further studies are warranted. Taken together, some pharmaceuticals can possibly be empirically characterized as not embryo-fetal toxicants based on negative results from alternative assays and EFD study with one animal species instead of EFD studies with 


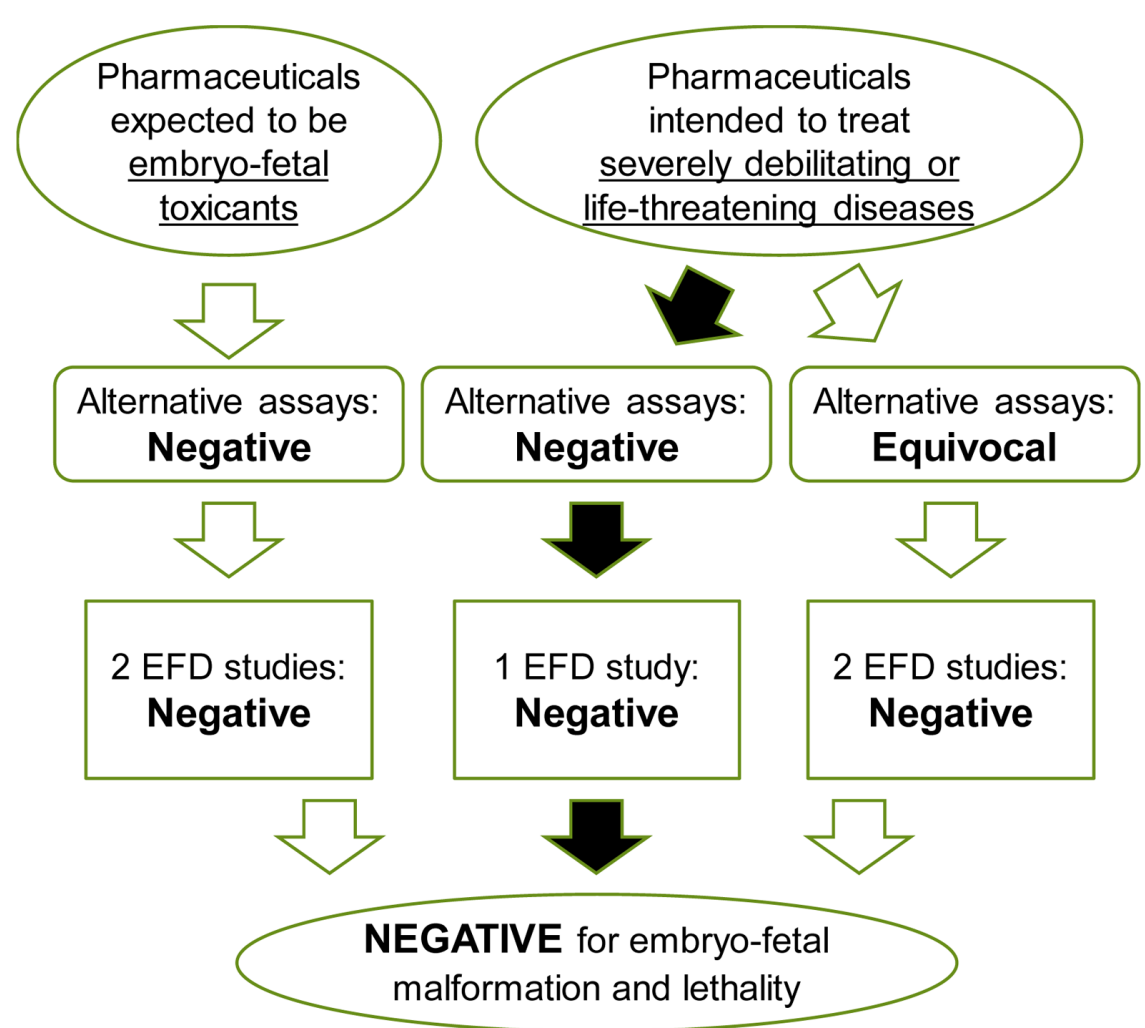

Fig. 1. Required embryo-fetal developmental (EFD) studies to show "negative" for embryo-fetal malformation and lethality in each scenario. Note that if some pharmaceuticals can be applicable to the scenario "pharmaceuticals intended for treating severely debilitating or life-threatening diseases", there is a chance to show negative for embryo-fetal malformation and lethality with only one EFD study instead of two. In this case, alternative assays must predict negative results of one animal species (rodents or rabbits) other than animal species used in EFD study (rabbits or rodents, respectively). Two EFD studies: one EFD study with rodents and another EFD study with rabbits.

two animal species. Therefore, validating and evaluating the results of alternative assays compared with those of in vivo EFD studies may be required in the future, and this can be another topic of regulatory science studies. In any case, some alternative assays are still in development, and highly qualified assays may thus be invented in the near future and should be evaluated by regulatory or other authorities using science-based knowledge.

\section{Conclusion and Future Perspective}

In this review article I have suggested that regulatory science studies have yet to be performed to evaluate the EFD toxicity of pharmaceuticals for human use. I have outlined several topics in regulatory science studies; however, there are many others that need to be addressed now or in the future. Nevertheless, the research topics described here would greatly contribute to the $3 \mathrm{R}$ principles without compromising the safety of women of childbearing potential, pregnant women, and their children. In addition, it would be important to clarify the number of patients with each disease and the number of women of childbearing potential in those patient populations, and to analyze data to generate a new approach to embryo-fetal toxicity evaluation. Lastly, as the effects of pharmaceuticals on epigenetics in the mam- malian fetus and offspring have not been studied so far, detection of adverse epigenetic changes due to the use of pharmaceuticals in pregnant women may be a next step in realizing safe pharmaceuticals.

\section{Conflict of Interest}

In performing this research, the author did not receive any specific grant from funding agencies in the public, commercial, or not-for-profit sectors. The author declares that he has no conflicts of interest.

\section{References}

1. Ridings, J. E. 2013. The thalidomide disaster, lessons from the past. Methods Mol. Biol. 947: 575-586. [Medline] [CrossRef]

2. Russell, W. M. S. and Burch, R. L. 1992. The Principles of Humane Experimental Technique. Special Edition Universities Federation for Animal Welfare (UFAW) Wheathampstead.

3. International Council on Harmonisation S5 (R3) 2020. Guideline on reproductive toxicology: Detection of toxicity to reproduction for human pharmaceuticals. https://database.ich.org/sites/default/files/S5-R3_Step4_ Guideline_2020_0218_1.pdf [accessed May 22, 2020].

4. Theunissen, P.T., Beken, S., Beyer, B. K., Breslin, W. J., Cappon, G. D., Chen, C. L., Chmielewski, G., De Schaepdrijver, 
L., Enright, B., Foreman, J. E., Harrouk, W., Hew, K. W., Hoberman, A. M., Hui, J. Y., Knudsen, T. B., Laffan, S. B., Makris, S. L., Martin, M., McNerney, M. E., Siezen, C. L., Stanislaus, D. J., Stewart, J., Thompson, K. E., Tornesi, B., Van der Laan, J. W., Weinbauer, G. F., Wood, S. and Piersma, A. H. 2016. Comparison of rat and rabbit embryo-fetal developmental toxicity data for 379 pharmaceuticals: on the nature and severity of developmental effects. Crit. Rev. Toxicol. 46: 900-910. [Medline] [CrossRef]

5. Necitumumab (Genetical Recombination) Portrazza ${ }^{\circledR}$ injection package insert. https://www.pmda.go.jp/Pmda Search/iyakuDetail/ResultDataSetPDF/530191_4291448A102 6_1_02 [accessed May 22, 2020].

6. Lorlatinib. Lorbrena ${ }^{\circledR}$ tablets package insert. https://www. pmda.go.jp/PmdaSearch/iyakuDetail/ResultDataSetPDF/671 450_4291055F1020_1_03 [accessed May 22, 2020].

7. Favipiravir. PMDA Review for Avigan ${ }^{\circledR}$. https://www.pmda. go.jp/files/000210319.pdf [accessed May 22, 2020].

8. Azilsartan. Azilva ${ }^{\circledR}$ tablets package insert. https://www. pmda.go.jp/PmdaSearch/iyakuDetail/ResultDataSetPDF/400 256_2149048F1022_1_13 [accessed May 22, 2020].

9. Candesartan Cilexetil Blopress ${ }^{\circledR}$ tablets package insert. https://www.pmda.go.jp/PmdaSearch/iyakuDetail/Result DataSetPDF/400061_2149040F1026_2_02 [accessed May 22, 2020].

10. Irbesartan. Irbetan ${ }^{\circledR}$ tablets package insert. https://www. pmda.go.jp/PmdaSearch/iyakuDetail/ResultDataSetPDF/343 018_2149046F1031_2_03 [accessed May 22, 2020].

11. Losartan Potassium Nu-Lotan ${ }^{\circledR}$ tablets package insert. https://www.pmda.go.jp/PmdaSearch/iyakuDetail/Result DataSetPDF/170050_2149039F1031_2_12 [accessed May 22, 2020].

12. Olmesartan Medoxomil Olmetec ${ }^{\circledR}$ OD tablets package insert. https://www.pmda.go.jp/PmdaSearch/iyakuDetail/Result DataSetPDF/430574_2149044F5020_1_04 [accessed May 22, 2020].

13. Telmisartan. Micardis ${ }^{\circledR}$ tablets package insert. https://www. pmda.go.jp/PmdaSearch/iyakuDetail/ResultDataSetPDF/650 168_2149042F1025_1_23 [accessed May 22, 2020].

14. Valsartan. Diovan ${ }^{\circledR}$ OD tablets package insert. https://www. pmda.go.jp/PmdaSearch/iyakuDetail/ResultDataSetPDF/300 242_2149041F5026_1_11 [accessed May 22, 2020].

15. Atezolizumab (Genetical Recombination) Tecentriq ${ }^{\circledR}$ package insert. https://www.pmda.go.jp/PmdaSearch/iyakuDetail/Re sultDataSetPDF/450045_4291441A1024_1_09 [accessed May 22, 2020].

16. Amlodipine Besilate Norvasc ${ }^{\circledR}$ tablets package insert. https:// www.pmda.go.jp/PmdaSearch/iyakuDetail/ResultDataSetP DF/671450_2171022F1029_2_15 [accessed May 22, 2020].

17. Azelnidipine. Calblock ${ }^{\circledR}$ tablets package insert. https://www. pmda.go.jp/PmdaSearch/iyakuDetail/ResultDataSetPDF/430 574_2149043F1020_2_12 [accessed May 22, 2020].

18. Benidipine Hydrochloride Coniel ${ }^{\circledR}$ tablets package insert. https://www.pmda.go.jp/PmdaSearch/iyakuDetail/Result DataSetPDF/230124_2171021F1024_1_19 [accessed May 22, 2020].

19. Cilnidipine. Atelec ${ }^{\circledR}$ tablets package insert. https://www. pmda.go.jp/PmdaSearch/iyakuDetail/ResultDataSetPDF/111 890_2149037F1032_2_06 [accessed May 22, 2020].

20. Diltiazem Hydrochloride Herbesser ${ }^{\circledR}$ tablets package insert. https://www.pmda.go.jp/PmdaSearch/iyakuDetail/Result DataSetPDF/400315_2171006F1224_1_10 [accessed May 22, 2020].

21. Manidipine Hydrochloride Calslot ${ }^{\circledR}$ tablets package insert. https://www.pmda.go.jp/PmdaSearch/iyakuDetail/Result DataSetPDF/400061_2149027F1020_2_01 [accessed May 22, 2020].

22. Nicardipine Hydrochloride Perdipine ${ }^{\circledR}$ tablets package insert. https://www.pmda.go.jp/PmdaSearch/iyakuDetail/Re
sultDataSetPDF/171911_2149019B1059_2_01 [accessed May 22, 2020].

23. Nifedipine. Adalat ${ }^{\circledR}$ capsule package insert. https://www. pmda.go.jp/PmdaSearch/iyakuDetail/ResultDataSetPDF/630 004_2171014M1104_1_14 [accessed May 22, 2020].

24. Foundation for Promotion of Cancer Research Cancer Statistics in Japan. 2018, https://ganjoho.jp/data/reg_stat/ statistics/brochure/2018/cancer_statistics_2018_fig_E.pdf [accessed May 22, 2020].

25. Ngu, S. F. and Ngan, H. Y. S. 2016. Chemotherapy in pregnancy. Best Pract. Res. Clin. Obstet. Gynaecol. 33: 86-101. [Medline] [CrossRef]

26. Andrews, P. A., Blanset, D., Costa, P. L. , Green, M., Green, M. L., Jacobs, A., Kadaba, R., Lebron, J. A., Mattson, B., McNerney, M. E., Minck, D., Oliveira, L. C., Theunissen, P. T. and DeGeorge, J. J. 2019. Analysis of exposure margins in developmental toxicity studies for detection of human teratogens. Regul Toxicol Pharmacol. 105: 62-68. [Medline] [CrossRef]

27. Ito, T., Ando, H., Suzuki, T., Ogura, T., Hotta, K., Imamura, Y., Yamaguchi, Y. and Handa, H. 2010. Identification of a primary target of thalidomide teratogenicity. Science 327: 1345-1350. [Medline] [CrossRef]

28. Lopez-Girona, A., Mendy, D., Ito, T., Miller, K., Gandhi, A. K., Kang, J., Karasawa, S., Carmel, G., Jackson, P., Abbasian, M., Mahmoudi, A., Cathers, B., Rychak, E., Gaidarova, S., Chen, R., Schafer, P. H., Handa, H., Daniel, T. O., Evans, J. F. and Chopra, R. 2012. Cereblon is a direct protein target for immunomodulatory and antiproliferative activities of lenalidomide and pomalidomide. Leukemia 26: 2326-2335. [Medline] [CrossRef]

29. Mahony, C., Erskine, L., Niven, J., Greig, N. H., Figg, W. D. and Vargesson, N. 2013. Pomalidomide is nonteratogenic in chicken and zebrafish embryos and nonneurotoxic in vitro. Proc. Natl. Acad. Sci. USA 110: 12703-12708. [Medline] [CrossRef]

30. Pomalidomide. USFDA full prescribing information. https://www.accessdata.fda.gov/drugsatfda_docs/ label/2017/204026s019lbl.pdf [accessed May 22, 2020].

31. Lenalidomide. USFDA full prescribing information. https://www.accessdata.fda.gov/drugsatfda_docs/ label/2012/021880s028lbl.pdf [accessed May 22, 2020].

32. Jo, S., Lee, K. H., Song, S., Jung, Y. K. and Park, C. S. 2005. Identification and functional characterization of cereblon as a binding protein for large-conductance calcium-activated potassium channel in rat brain. J. Neurochem. 94: 1212-1224. [Medline] [CrossRef]

33. Higgins, J. J., Hao, J., Kosofsky, B. E. and Rajadhyaksha, A. M. 2008. Dysregulation of large-conductance Ca2+-activated $\mathrm{K}+$ channel expression in nonsyndromal mental retardation due to a cereblon p.R419X mutation. Neurogenetics 9: 219-223. [Medline] [CrossRef]

34. Tebipenem pivoxil. Summary technical documentation to Regulatory Agency in Japan. https://www.pmda.go.jp/ drugs/2000/g000911/43ctdp_1-343.pdf [accessed May 22, 2020].

35. Amoxicillin hydrate. Summary technical documentation to Regulatory Agency in Japan. https://www.pmda.go.jp/ drugs/2000/g000908/46ctdp_1-201.pdf [accessed May 22, 2020].

36. Azithromycin hydrate. Summary technical documentation to Regulatory Agency in Japan. https://www.pmda.go.jp/ drugs/2000/g000309/19ctdp_1-426.pdf [accessed May 22, 2020].

37. Cefazolin Sodium Cefamezin ${ }^{\circledR} \alpha$ interview form. https:// www.info.pmda.go.jp/go/interview/4/171911_613240 1G1041_1_CMK_2F.pdf [accessed May 22, 2020].

38. Clofazimine. Lampren ${ }^{\circledR}$ capsules interview form. https:// www.info.pmda.go.jp/go/interview/3/270428_623900 
1M1022_3_L04_1F.pdf [accessed May 22, 2020].

39. Ertapenem sodium. USFDA Pharm/tox Review. https:// www.accessdata.fda.gov/drugsatfda_docs/nda/2001/21337_ Invanz_pharmr.pdf [accessed May 22, 2020].

40. Brehm, M. A., Shultz, L. D. and Greiner, D. L. 2010. Humanized mouse models to study human diseases. Curr. Opin. Endocrinol. Diabetes Obes. 17: 120-125. [Medline] [CrossRef]

41. Heuts, F. and Nagy, N. 2017. Mice with reconstituted human immune system components as a tool to study Immune cell interactions in EBV infection. Methods Mol. Biol. 1532: 229-240. [Medline] [CrossRef]

42. Walsh, N. C., Kenney, L. L., Jangalwe, S., Aryee, K. E., Greiner, D. L., Brehm, M. A. and Shultz, L. D. 2017. Humanized Mouse Models of Clinical Disease. Annu. Rev. Pathol. 12: 187-215. [Medline] [CrossRef]

43. Gammelgaard, O. L., Terp, M. G., Preiss, B. and Ditzel, H.
J. 2018. Human cancer evolution in the context of a human immune system in mice. Mol. Oncol. 12: 1797-1810. [Medline] [CrossRef]

44. Li, Y. and Di Santo, J. P. 2019. Modeling infectious diseases in mice with a "Humanized" immune system. Microbiol. Spectr. 7: 10.1128/microbiolspec.BAI-0019-2019. [Medline]

45. Rios-Doria, J., Stevens, C., Maddage, C., Lasky, K. and Koblish, H. K. 2020. Characterization of human cancer xenografts in humanized mice. J. Immunother. Cancer 8: e000416. [Medline] [CrossRef]

46. International Council on Harmonisation M3(R2). Guidance on Nonclinical Safety Studies for the Conduct of Human Clinical Trials and Marketing Authorization for Pharmaceuticals (2009) together with ICH M3(R2). Questions \& answers. 2012. https://database.ich.org/sites/default/files/ M3_R2_Q\%26As_R2_Q\%26As_0.pdf [accessed May 22, 2020]. 